\title{
Destructive Intergenerational Altruism
}

\author{
Geir B. Asheim, Frikk Nesje
}

\begin{abstract}
Are the probable future negative effects of climate change an argument for decreasing the discount rate to promote the interests of future generations? The anal$y$ sis of the present paper suggests that such stronger intergenerational altruism might undermine future well-being if not complemented by collective climate action. In the standard one-sector model of economic growth normatively attractive outcomes will be implemented if each generation has sufficient altruism for its descendants. This conclusion is radically changed in a two-sector model where one form of capital is more productive than the other but leads to negative atmospheric externalities. In fact, the model shows that, if each dynasty is trying to get ahead in a world threatened by climate change by increasing its intergenerational altruism, then long-term wellbeing will be seriously undermined.
\end{abstract}

JEL Codes: D63, D64, D71, Q01, Q54

Keywords: Climate change, Intergenerational altruism

IN MANY COUNTRIES, environmentalists argue for a lower discount rate to promote the interests of future generations, in particular in face of the probable future negative consequences of climate change. ${ }^{1}$ This would allow for more investments to be real-

Geir B. Asheim (corresponding author) is at the Department of Economics, University of Oslo, PO Box 1095 Blindern, N-0317 Oslo, Norway (g.b.asheim@econ.uio.no). Frikk Nesje is at the Department of Economics, University of Oslo, PO Box 1095 Blindern, N-0317 Oslo, Norway (frikk.nesje@econ.uio.no). This paper has previously been circulated under the title "Competitive Intergenerational Altruism." We are grateful for helpful discussions with Ivar Ekeland, Nicolai Ellingsen, Bård Harstad, Paolo Piacquadio, Armon Rezai, and Alessia Russo and constructive comments by the editor and two reviewers, as well as seminar participants in Alba di Canazei, Amsterdam, Bergen, Brighton, Oslo, Santa Barbara, St. Petersburg, and Toulouse. The paper is also part of the research activities at the Centre for the Study of Equality, Social Organization, and Performance (ESOP) at the Department of Economics at the University of Oslo. ESOP is supported by the Research Council of Norway through its Centres of Excellence funding scheme, project number 179552.

1. For example, the US environmental organization the Sierra Club recommends the "use of a discount rate less than the opportunity cost of capital" in order to mitigate environmental im-

Received December 16, 2015; Accepted May 23, 2016; Published online November 9, 2016.

JAERE, volume 3, number 4. (C) 2016 by The Association of Environmental and Resource Economists. All rights reserved. 2333-5955/2016/0304-0006\$10.00 http://dx.doi.org/10.1086/688584 
ized in the public sector and also in the private sector, if private investors were induced to assign more weight on the future, thereby compensating for future adverse climate effects. This paper illustrates that such an advice might be counterproductive, if not combined with collective climate action. In more general terms, the aim of the paper is to construct a simple model where increased intergenerational altruism has serious counterproductive consequences in a setting with atmospheric externalities.

The basic model of economic growth is the one-sector model where capital and an exogenous amount of labor are used to produce output, which is split into consumption and capital investment. If interpreted as a model where nonoverlapping generations follow each other in sequence, and the welfare of any generation depends linearly on the utility that it derives from its consumption and the welfare of the next, then the behavior will be as if the economy is following a discounted utilitarian optimum. If the altruism for the next generation is sufficiently strong compared to the net productivity of capital, then the realized stream has nice properties: it is increasing in time and converges to a steady state with constant per capita consumption.

This model can be interpreted as representing an economy divided into dynasties (families or tribes) where the members of any generation care for the welfare of the immediate descendants in their own dynasty. Since the welfare of the immediate descendants depends on the welfare of their descendants and so forth, the interests of the members of later generations are indirectly taken into account. If this model describes the technological opportunities and the preferences of real economies, then the question of implementing an equitable intergenerational distribution is reduced to each generation having sufficient altruism for the immediate descendants of their own dynasty.

Suppose that instead there are two different kinds of capital, where one form of capital is more productive, but where the aggregate stock of this form of capital produces negative atmospheric externalities that reduce utility for all dynasties. If each dynasty, in this setting, seeks to protect its immediate descendants against the effect of climate change, then reducing its own greenhouse gas emissions by accumulating the less productive asset may not be a productive line of action. Rather, it is reasonable that a dynasty will choose to accumulate the more productive asset, thereby ensuring the next generation of its own dynasty a better start in life at the cost of all other dynasties through increasing greenhouse gas emissions. Consequently, given the external effects of greenhouse gas emissions, dynasties taking care of their descendants do not necessarily contribute to solving the efficiency problem that climate change poses, but may add gravity to the problem.

pacts (Sierra Club 2016). In Norway, WWF and the Norwegian NGO, Bellona, among others, argue in favor of lower discount rates to promote the interests of future generations (Government of Norway 2016). 
Efficient policies at a societal level, on the other hand, require that the superior productiveness is weighted against the atmospheric externalities that such capital leads to. The nice properties of the one-sector model might therefore disappear when the asset that the dynasties choose to accumulate is a public bad. This paper illustrates that intergenerational altruism can indeed be destructive, since caring for future generations may seriously undermine future well-being at the aggregate level.

In this paper we contribute to the literature by providing a clear representation of this underlying mechanism. Furthermore, we evaluate the consequences of intergenerational altruism in the settings without and with atmospheric externalities by applying normative criteria for intergenerational equity.

There are models that include both intergenerational altruism and uninternalized environmental externalities, with varying results on whether intergenerational altruism can to some extent substitute for appropriate environmental policy. ${ }^{2}$ For example, Karp (forthcoming) shows, in an overlapping generations model, that cooperation is relatively more important than intergenerational altruism for linear equilibrium public good provision. Relatedly, based on the assumption from Foley (2008) that each agent's contribution to the aggregate is negligible, Rezai, Foley, and Taylor (2012) show (in their table 2) that a reduction of the discount rate may lead to increased levels of atmospheric carbon and a larger capital stock. The economic intuition is that a lower discount rate leads to higher saving if the externality is not internalized (i.e., if the path of emissions is taken as exogenous in the dynasties' decision). This is in line with the main insight from the present paper, namely, that stronger concern for future generations need not be an effective substitute for collective climate action.

The approach of Jouvet, Michel, and Vidal (2000) is different. Using an overlapping generations model, they characterize conditions in which altruistic agents voluntarily contribute to environmental quality. Provided positive bequest, the "threshold triggering positive contributions [to pollution abatement is] lower, the higher the degree of intergenerational altruism" (Michel, Thibault, and Vidal 2006, 1102). The competitive equilibrium is inefficient, though, as the environmental externality of capital accumulation and the social willingness to pay for pollution abatement are not fully internalized. This finding relates to the early contribution to the literature by Howarth and Norgaard (1995), where it is highlighted that there might be a need for collective action, since altruistic agents do not necessarily internalize all externalities. Contrary to

2. Farzin (1984) and Asheim (2008) point out how more weight on the future through a lower discount rate might accelerate the extraction of natural resources by reducing the cost of capital. This might even lead to lower long-run well-being. This mechanism is similar to the one considered here as the lower discount rate promotes capital accumulation, but also different as externalities play no role. 
the above-mentioned papers, we show how increased intergenerational altruism can be seriously counterproductive with global (atmospheric) externalities.

In another related study, John and Pecchenino $(1994,1393)$ illustrate consequences of short-lived agents' decisions "concerning the accumulation of capital and the provision of a public good, environmental quality" in an overlapping generations model. They focus on how externalities affect utility through consumption, while our interest is in the transmission of these effects through capital accumulation.

The paper is organized as follows. In section 1, we recapitulate the Ramsey model, on which the analysis is based, and show the consequences of different criteria of intergenerational equity in the setting of this model. Furthermore, we show how streams promoted by all these criteria can be implemented by sufficient intergenerational altruism, even in a world decentralized into dynasties. In section 2, we consider a two-sector model, with two capital goods: "brown" and "green" capital. In line with what has been presented earlier in the introduction, we assume that "brown" capital is more productive but is also a public bad in the sense of leading to atmospheric externalities that affect all dynasties in the same manner. In section 3, we show that streams implemented in a world decentralized into dynasties cannot be endorsed by criteria of intergenerational equity, if intergenerational altruism is sufficiently strong. In section 4, we investigate the scope of collective climate action. In section 5 , we discuss whether main results are robust to relaxing some core assumptions. In section 6 we conclude.

There are four appendices. Appendix A presents in more detail the different criteria of intergenerational equity. Appendix B establishes the existence of the equilibrium of the game that the altruistic dynasties will play and shows the properties of this equilibrium, thereby proving the results of section 2. Appendix $\mathrm{C}$ establishes the existence of an efficient maximin stream that facilitates the normative analysis of section 3. Appendix $\mathrm{D}$ establishes, in a special case, the highest possible productivity difference between "brown" and "green" capital consistent with short-run efficiency in all periods in the case where only "green" capital is accumulated, thereby facilitating the analysis of section 4 .

\section{RAMSEY MODEL}

Assume that the technology is given by an increasing, strictly concave, and twice continuously differentiable production function $f: \mathbb{R}_{+} \rightarrow \mathbb{R}_{+}$, satisfying $f(0)=0$, $\lim _{k \rightarrow \infty} f(k)=\bar{c}<\infty$ (implying that $\lim _{k \rightarrow \infty} f^{\prime}(k)=0$ ) and $\lim _{k \rightarrow 0} f^{\prime}(k)=\infty$. Labor is constant and normalized to 1 , implying that $f(k)=F(k, 1)$ for all $k \in$ $\mathbb{R}_{+}$, where $F: \mathbb{R}_{+} \times \mathbb{R}_{+} \rightarrow \mathbb{R}_{+}$is a linearly homogeneous production function of capital and labor.

A consumption stream ${ }_{1} c=\left(c_{1}, c_{2}, \ldots\right) \geq 0$ is feasible given an initial capital stock $k>0$ if there exists a capital stream ${ }_{0} k=\left(k_{0}, k_{1}, k_{2} \ldots\right) \geq 0$ such that $k_{0}=k$ and

$$
c_{t}+k_{t}=k_{t-1}+f\left(k_{t-1}\right)
$$


for all $t \in \mathbb{N}^{3}$ Such a technology is referred to as a Ramsey model. Denote by

$$
K(k)=\left\{{ }_{0} k: k_{0}=k \text { and } 0 \leq k_{t} \leq k_{t-1}+f\left(k_{t-1}\right) \text { for all } t \in \mathbb{N}\right\}
$$

the set of feasible capital streams. Write $\mathcal{K}=\cup_{k \in \mathbb{R}_{+}} K(k)$.

Define

$$
\mathbf{c}\left({ }_{0} k\right)=\left(k_{0}+f\left(k_{0}\right)-k_{1}, k_{1}+f\left(k_{1}\right)-k_{2}, \ldots, k_{t-1}+f\left(k_{t-1}\right)-k_{t}, \ldots\right)
$$

as the consumption stream that is associated with ${ }_{0} k$, and denote by

$$
C(k)=\left\{{ }_{1} c: \text { there is }{ }_{0} k \in K(k) \text { such that }{ }_{1} c=c\left({ }_{0} k\right)\right\}
$$

the set of feasible consumption streams. Say that ${ }_{1} c \in C(k)$ is efficient if there is no ${ }_{1} \tilde{c} \in C(k)$ such that ${ }_{1} \tilde{c}>{ }_{1} c$.

Map consumption $c$ into utility by an increasing, strictly concave, and continuously differentiable utility function $u: \mathbb{R}_{+} \rightarrow \mathbb{R}$, satisfying $u(0)=0$ and $\lim _{c \rightarrow 0} u^{\prime}(c)=\infty$. Define $\mathbf{u}\left({ }_{1} c\right)=\left(u\left(c_{1}\right), u\left(c_{2}\right), \ldots, u\left(c_{t}\right) \ldots\right)$ and denote by

$$
U(k)=\left\{{ }_{1} u: \text { there is }{ }_{1} c \in C(k) \text { such that }{ }_{1} u=\mathbf{u}\left({ }_{1} c\right)\right\}
$$

the set of feasible utility streams. Write $\mathcal{U}=\cup_{k \in \mathbb{R}_{+}} U(k)$.

Let labor be uniformly distributed over a continuum of dynasties $i$ on the unit interval $[0,1]$. Let, at each time $t, \mathbf{k}_{t}:[0,1] \rightarrow \mathbb{R}_{+}$be the profile of capital ownership across the dynasties and $\mathbf{c}_{t}:[0,1] \rightarrow \mathbb{R}_{+}$the profile of consumption. Assume that $\mathbf{k}_{t}$ and $\boldsymbol{c}_{t}$ are Lebesgue measurable and sum up to aggregate capital and consumption:

$$
k_{t}=\int_{0}^{1} \mathbf{k}_{t}(i) d i, c_{t}=\int_{0}^{1} \mathbf{c}_{t}(i) d i .
$$

At each time $t \in \mathbb{N}$, the budget constraint for each dynasty $i \in[0,1]$ is given by:

$$
\mathbf{c}_{t}(i)+\mathbf{k}_{t}(i)=\mathbf{k}_{t-1}(i)+f\left(k_{t-1}\right)+f^{\prime}\left(k_{t-1}\right)\left(\mathbf{k}_{t-1}(i)-k_{t-1}\right),
$$

where the terms $f\left(k_{t-1}\right)+f^{\prime}\left(k_{t-1}\right)\left(\mathbf{k}_{t-1}(i)-k_{t-1}\right)$ equal the value of the marginal products of the capital and labor held by dynasty $i$ at time $t-1$. $^{5}$

3. For two vectors $x$ and $y$, write $x \geq y$ if and only if $x_{t} \geq y_{t}$ for all $t, x>y$ if and only if $x \geq y$ and $x \neq y$, and $x \gg y$ if and only if $x_{t}>y_{t}$ for all $t$.

4. The normalization $u(0)=0$ will become important in sec. 2 where the effect of atmospheric externalities enters multiplicatively.

5. To derive eq. (2), note that, for dynasty $i$, the value of the marginal products at time $t$ equals:

$$
F_{1}\left(k_{t}, 1\right) \cdot \mathbf{k}_{t}(i)+F_{2}\left(k_{t}, l\right) \cdot 1=F\left(k_{t}, 1\right)+F_{1}\left(k_{t}, 1\right)\left(\mathbf{k}_{t}(i)-k_{t}\right)
$$


Assume that the present member of dynasty $i$ cares for the descendants in the same dynasty, but not for descendants in other dynasties. ${ }^{6}$ In particular, the preferences of each dynasty is assumed to be represented by the nonpaternalistic altruistic (NPA) welfare function $w_{\alpha}: \mathcal{U} \rightarrow \mathbb{R}$ defined by:

$$
w_{\alpha}\left({ }_{1} u\right)=(1-\alpha) \sum_{t=0}^{\infty} \alpha^{t} u_{t+1},
$$

where $\alpha \in(0,1)$ is the per generation factor used to discount future utilities. It follows from the assumptions on $f$ and $u$ that $w_{\alpha}$ is well defined on $\mathcal{U}$. The altruism is nonpaternalistic in the terminology of Ray (1987), as the welfare of generation $t$ is derived from its own consumption and the welfare of generation $t+1:^{7}$

$$
w_{\alpha}\left({ }_{t} u\right)=(1-\alpha) u_{t}+\alpha w_{\alpha}\left(_{t+1} u\right) .
$$

Hence, we assume that the dynasties have the same utility function $u$ and the same altruistic welfare function $w_{\alpha}$. If, in addition, the profile of initial ownership to capital is assumed to be uniform, so that $\mathbf{k}_{0}(i)=k$ for all $i \in[0,1]$, the dynasties will behave in the same manner, implying that it holds for each $t \in \mathbb{N}$ that $\mathbf{k}_{t}(i)=k_{t}$ for all $i \in$ $[0,1]$ so that equation (2) simplifies to equation (1). Letting a (representative) dynasty maximize $w_{\alpha}\left({ }_{1} u\right)$ over all ${ }_{1} u \in U(k)$ leads to a first-best solution. This first-best solution can be implemented as a unique equilibrium in the decentralized economy with each dynasty having zero measure, since $u$ is strictly concave. Hence, we may perform the analysis of this section by considering a representative dynasty. ${ }^{8}$

The NPA welfare function $v_{\alpha}: \mathcal{K} \rightarrow \mathbb{R}$ defined over capital streams is given by:

$$
v_{\alpha}\left({ }_{0} k\right)=w_{\alpha}\left(\mathbf{u}\left(\mathbf{c}\left({ }_{0} k\right)\right)\right)=(1-\alpha) \sum_{t=0}^{\infty} \alpha^{t} u\left(k_{t}+f\left(k_{t}\right)-k_{t+1}\right),
$$

with $\alpha \in(0,1)$. Say that ${ }_{0} k \in K(k)$ is an NPA optimum if

$$
v_{\alpha}\left({ }_{0} k\right) \geq v_{\alpha}\left({ }_{0} \tilde{k}\right) \text { for all }{ }_{0} \tilde{k} \in K(k) \text {. }
$$

by using the property that $F$ is homogeneous of degree 1 . We obtain $f\left(k_{t}\right)+f^{\prime}\left(k_{t}\right)\left(\mathbf{k}_{t}(i)-k_{t}\right)$ by substituting $f\left(k_{t}\right)$ for $F\left(k_{t}, 1\right)$ and $f^{\prime}\left(k_{t}\right)$ for $F_{1}\left(k_{t}, 1\right)$.

6. Altruism for the descendants of all dynasties leads to a severe public good problem, thus necessitating collective action; see sec. 5 .

7. The NPA welfare function as given by (3) is in the family of time-discounted utilitarian (TDU) welfare functions, presented in appendix A. As shown by Saez-Marti and Weibull (2005), there are NPA welfare functions that are not in this family if the nonpaternalistic altruism extends beyond the next generation.

8. We have introduced a decentralized model with dynasties already in this section to prepare for the analysis of the decentralized equilibrium of sec. 2. Moreover, we refer to the decentralized model of this section when discussing altruism for the descendants of all dynasties in sec. 5 . 
Before characterizing the optimum, we need to define the modified golden rule. Define $k_{\infty}:(0,1) \rightarrow \mathbb{R}_{+}$by, for all $\alpha \in(0,1)$,

$$
\alpha\left(1+f^{\prime}\left(k_{\infty}(\alpha)\right)\right)=1 .
$$

It follows from the properties of $f$ that $k_{\infty}$ is well defined, continuous, and increasing, with $\lim _{\alpha \rightarrow 0} k_{\infty}(\alpha)=0$ and $\lim _{\alpha \rightarrow 1} k_{\infty}(\alpha)=\infty$. For given $\alpha \in(0,1), k_{\infty}(\alpha)$ is the capital stock corresponding to the modified golden rule.

By Beals and Koopmans (1969), we have the following result: ${ }^{9}$

Proposition 1: There is a unique NPA optimum, $\hat{k}(k)$, with associated NPA optimal consumption stream $\hat{c}(k)=\boldsymbol{c}(\hat{k}(k))$. Furthermore, $\hat{k}(k)$ is strictly monotone in time, with $\lim _{t \rightarrow \infty} \hat{k}_{t}(k)=k_{\infty}(\alpha)$, and $\hat{c}(k)$ is efficient, with $\lim _{t \rightarrow \infty} \hat{c}_{t}(k)=f\left(k_{\infty}(\alpha)\right)$.

The following is a straightforward implication of proposition 1:

Corollary 1: Let $\alpha^{*}=1 /\left(1+f^{\prime}(k)\right)$.

(a) If $\alpha \in\left(\alpha^{*}, 1\right)$, then the NPA optimum, $\hat{k}(k)$, is increasing in time.

(b) If $\alpha=\alpha^{*}$, then the NPA optimum, $\hat{k}(k)$, is constant in time.

(c) If $\alpha \in\left(0, \alpha^{*}\right)$, then the NPA optimum, $\hat{k}(k)$, is decreasing in time.

The central question of this paper is whether nonpaternalistic intergenerational altruism leads a normatively attractive outcome. Normative criteria-derived from axiomatic analysis and designed to balance the interests of present and future generations from an impartial perspective-can be used to evaluate the normative attractiveness of outcomes. In appendix A, several such criteria are considered.

A fundamental criterion is Suppes-Sen maximality (Suppes 1966; Sen 1970), which combines efficiency in the sense of being sensitive to the interests of each generation (the Strong Pareto axiom) with equal treatment in the sense of being insensitive to all finite reorderings of the consumption stream (the Finite Anonymity axiom). ${ }^{10}$

The Calvo criterion (Calvo 1978) maximizes the infimum of the altruistic welfare of all generations. Calvo (1978) shows that there exists a unique and time-consistent Calvo optimum in the Ramsey model.

9. The result that also $\hat{c}(k)$ is monotone in time is left out of proposition 1 to make the statement parallel to that of proposition 2, where the consumption stream need not be monotone.

10. The Suppes-Sen criterion is incomplete as it compares only pairs that dominate each other after some finite reordering. It follows from the Lauwers-Zame (Zame 2007; Lauwers 2010) impossibility result (see Asheim 2010, sec. 3.2) that no explicitly describable complete criterion can satisfy both the Strong Pareto and Finite Anonymity axioms. 
Appendix A also presents the sustainable discounted utilitarian (SDU) criterion (Asheim and Mitra 2010) and the rank-discounted utilitarian (RDU) criterion (Zuber and Asheim 2012). In these criteria, utility $u_{t}$ as an indicator of well-being for each generation $t$ is turned into generalized utility $v\left(u_{t}\right)$ by a continuous and increasing function $v: \mathbb{R}_{+} \rightarrow \mathbb{R}$. Both SDU and RDU discount future generalized utility by a discount factor $\beta \in(0,1)$, only if the future is better off than the present, ${ }^{11}$ thereby trading-off current sacrifice and future gain. In this case, future's higher consumption is discounted because,

- at a higher level, added consumption contributes less to utility if the composite function $v \circ u$ is strictly concave, and

- being better off, its utility is assigned less weight since $\beta<1$.

Hence, if consumption is perfectly correlated with time, these criteria work as the ordinary time-discounted utilitarian (TDU) criterion which economists usually promote. The important difference is that, in the criteria of SDU and RDU, the future is discounted because priority is given to the worse-off earlier generations.

However, if the present is better off than the future, then priority shifts to the future. In this case, future utility is not discounted. Then the relative weights on future generations sum up to one, implying that zero relative weight remains for the present generation. ${ }^{12}$ The criteria of SDU and RDU can therefore capture the intuition that we should seek to assist future generations if they are worse off than us, while not having an unlimited obligation to save for their benefit if they turn out to be better off. Also, RDU is compatible with equal treatment of generations by being insensitive to all reorderings of the consumption stream (formally, RDU satisfies the Strong Anonymity axiom).

The next result makes the following main point: The NPA optimum-interpreted as the outcome implemented by maximizing dynasties with nonpaternalistic altruismis an outcome that can be endorsed by the Suppes-Sen, Calvo, SDU, and RDU criteria if and only if there is sufficient altruism.

Theorem 1: Let $\alpha^{*}=1 /\left(1+f^{\prime}(k)\right)$, and assume that all generations are nonpaternalistically altruistic according to equation (3). Then the implemented capital stream, $\hat{k}(k)$, with associated consumption stream, $\hat{c}(k)=\boldsymbol{c}(\hat{k}(k))$, is

11. In the case of RDU, this statement is exact only if all future generations are better off. For SDU, it is sufficient that the future, as aggregated by the SDU criterion, is better off.

12 . Note that SDU and RDU are numerically representable criteria. Under nonrepresentable undiscounted utilitarian criteria (see, e.g., Basu and Mitra 2007), it is possible to be sensitive for the interests of the present generation. 
- Suppes-Sen maximal,

- Calvo optimal, and

- SDU optimal and RDU optimal with $\beta=\alpha$ and $v(u)=u$,

if and only if $\alpha \geq \alpha^{*}$. Furthermore, long-term consumption, $\lim _{t \rightarrow \infty} \hat{c}_{t}(k)=$ $f\left(k_{\infty}(\alpha)\right)$, is increasing as a function of $\alpha$.

Hence, in the context of the Ramsey model, sufficient nonpaternalistic altruism leads to streams that can be justified by criteria that balance the interests of present and future generations from an impartial perspective (and, in the case of Suppes-Sen and RDU, treat generations equally). The result on Suppes-Sen maximality follows from Asheim, Buchholz, and Tungodden (2001, propositions 5-6 and example 2); this result requires only that the consumption stream is efficient and nondecreasing. The result on Calvo optimality follows from Calvo (1978, proposition 2), the result on SDU optimality follows from Asheim and Mitra (2010, theorem 2), while the result on RDU optimality follows from Zuber and Asheim (2012, proposition 10). The results on Calvo, SDU, and RDU optimality utilize the specific structure of the consumption stream obtained through the maximization of the NPA welfare function.

\section{ATMOSPHERIC EXTERNALITIES}

Assume now that there are two kinds of capital, brown and green capital, where brown capital is more productive than green capital, but where the aggregate stock of brown capital produces negative externalities that reduce utility for everyone by the same multiplicative factor. We refer to such negative effects as atmospheric externalities.

In this alternative version of the Ramsey model, a consumption $\operatorname{stream}_{1} c=\left(c_{1}, c_{2}, \ldots\right) \geq$ 0 is feasible given a pair of initial capital stocks $(b, g)>0$ if there exist streams of brown capital ${ }_{0} b=\left(b_{0}, b_{1}, b_{2} \ldots\right) \geq 0$ and green capital ${ }_{0} g=\left(g_{0}, g_{1}, g_{2} \ldots\right) \geq 0$ such that $\left(b_{0}, g_{0}\right)=(b, g)$ and

$$
c_{t}+b_{t}+g_{t}=b_{t-1}+g_{t-1}+f\left(b_{t-1}+(1-\gamma) g_{t-1}\right)
$$

for all $t \in \mathbb{N}$, where $\gamma \in(0,1)$ measures to what extent green capital is less productive. ${ }^{13}$

Let, as before, labor be uniformly distributed over a continuum of dynasties $i$ on the unit interval $[0,1]$. Let, at each time $t, \mathbf{b}_{t}:[0,1] \rightarrow \mathbb{R}_{+}$and $\mathbf{g}_{t}:[0,1] \rightarrow \mathbb{R}_{+}$be the pro-

13. In sec. 5, we interpret the two kinds of capital and argue that the assumption of perfect substitutability between brown and green capital comes without much loss of generality. There we also show how our results are robust to allowing for learning by doing in the accumulation and use of green capital, in spite of the assumption that $\gamma$ is constant. 
files of capital ownership across the dynasties and $c_{t}:[0,1] \rightarrow \mathbb{R}_{+}$the profile of consumption. Assume that $\mathbf{b}_{t}, \mathbf{g}_{t}$, and $\mathbf{c}_{t}$ are Lebesgue measurable and sum up to aggregate amounts of the two types of capital and aggregate consumption:

$$
b_{t}=\int_{0}^{1} \mathbf{b}_{t}(i) d i, g_{t}=\int_{0}^{1} \mathbf{g}_{t}(i) d i, c_{t}=\int_{0}^{1} \mathbf{c}_{t}(i) d i .
$$

At each time $t \in \mathbb{N}$, the budget constraint for each dynasty $i \in[0,1]$ is given by:

$$
\begin{aligned}
\mathbf{c}_{t}(i)+\mathbf{b}_{t}(i) & +\mathbf{g}_{t}(i)=\mathbf{b}_{t-1}(i)+\mathbf{g}_{t-1}(i)+f\left(b_{t-1}+(1-\gamma) g_{t-1}\right) \\
& +f^{\prime}\left(b_{t-1}+(1-\gamma) g_{t-1}\right)\left(\mathbf{b}_{t-1}(i)-b_{t-1}+(1-\gamma)\left(\mathbf{g}_{t-1}(i)-g_{t-1}\right)\right),
\end{aligned}
$$

where the terms

$f\left(b_{t-1}+(1-\gamma) g_{t-1}\right)+f^{\prime}\left(b_{t-1}+(1-\gamma) g_{t-1}\right)\left(\mathbf{b}_{t-1}(i)-b_{t-1}+(1-\gamma)\left(\mathbf{g}_{t-1}(i)-g_{t-1}\right)\right)$ equal the value of the marginal products of the capital and labor held by dynasty $i$ at time $t-1$.

Assume from now on that the map from consumption to utility for dynasty $i$ in generation $t$ depends on the aggregate amount of brown capital accumulated by generation $t-1$ :

$$
a\left(b_{t-1}\right) u\left(\mathbf{c}_{t}(i)\right)
$$

where the continuous and decreasing function $a: \mathbb{R}_{+} \rightarrow \mathbb{R}$, satisfying $a(0)=1$ and $\lim _{b \rightarrow \infty} a(b)=0$, captures the effect of the atmospheric externalities caused by brown capital. Refer to $a\left(b_{t-1}\right) u\left(\mathbf{c}_{t}(i)\right)$ as adjusted utility.

That atmospheric externalities depend on the stock of brown capital is of course a simplified and crude representation of the effects of economic activity on climate change. In reality, the flow of greenhouse gas emissions depends on the use of brown capital; it is not that the stock of greenhouse gases is a function of the stock of brown capital. Under such realistic modeling, the concentration of greenhouse gases in the atmosphere may increase even if the stock of brown capital remains constant. One might therefore claim that our modeling of climate change is conservative in the sense of understating the long-term effects of brown capital accumulation.

In the alternative model of this section each dynasty $i$ can invest in either brown $\mathbf{b}_{t}(i)$ or green $\mathbf{g}_{t}(i)$ capital. Since each dynasty is of zero weight, it is individually rational for each dynasty to invest in brown capital only, as this relaxes its budget constraint, while not influencing the aggregate stock of brown capital that adjusts its utility. However, efficient policies at a societal level require that the superior productiveness of brown capital is weighted against the atmospheric externalities that such capital leads to. In this section we model and analyze decentralized decision making, while turning to the opportunities for superior aggregate policies in the subsequent section 3 . 
As in the previous section we assume that the dynasties have the same utility function $u$ defined on consumption, and have same altruistic welfare function $w_{\alpha}$ defined by (3) on the set of feasible streams of adjusted utility. In particular, as before, we assume that the present member of dynasty $i$ cares for the descendants in the same dynasty, but not for descendants in other dynasties. Moreover, we assume that the dynasties are affected by the atmospheric externalities caused by brown capital in the same manner, as captured by the function $a$.

If, in addition, the profile of initial ownership to capital is assumed to be uniform, so that $\mathbf{b}_{0}(i)=b$ and $\mathbf{g}_{0}(i)=g$ for all $i \in[0,1]$, the dynasties will behave in the same manner. This implies that it holds for each $t \in \mathbb{N}$ that $\mathbf{b}_{t}(i)=b_{t}$ and $\mathbf{g}_{t}(i)=0$ for all $i \in[0,1]$ so that equation (5) simplifies to equation (4). Since $u$ is strictly concave, we may perform the analysis by considering a representative dynasty.

The analysis is simplified by considering the case where $b>0$ and $g=0$, so that in each period, also period 0 , there is a positive stock of brown capital only ${ }^{14}$ Under this assumption and taking into account that dynasties will choose to accumulate only brown capital, the set of (brown) capital streams and consumption streams as a function of the initial stock are the same as in the original Ramsey model analyzed in section $1: K(b)$ and $C(b)$, respectively.

In the model with atmospheric externalities, we cannot proceed by letting a representative dynasty maximize NPA welfare

$$
w_{\alpha}\left(\mathbf{u}\left(\mathbf{c}\left({ }_{0} b\right),{ }_{0} b\right)\right)=(1-\alpha) \sum_{t=0}^{\infty} \alpha^{t} a\left(b_{t}\right) u\left(b_{t}+f\left(b_{t}\right)-b_{t+1}\right)
$$

over all ${ }_{0} b \in K(b)$, where

$$
\mathbf{u}\left({ }_{1} c,{ }_{0} b\right)=\left(a\left(b_{0}\right) u\left(c_{1}\right), a\left(b_{1}\right) u\left(c_{2}\right), \ldots, a\left(b_{t-1}\right) u\left(c_{t}\right), \ldots\right)
$$

denotes the stream of adjusted utility. The reason is that the representative dynasty in this problem can partially internalize the external effects by accumulating less brown capital, even though it, by construction, is prevented from avoiding these external effects by accumulating green capital. ${ }^{15}$ Thus, the solution to this problem does not correspond to a decentralized equilibrium without collective action.

Instead, we must consider the case where the representative dynasty does not internalize the external effects of brown capital. Therefore, we let the representative dynasty maximize NPA welfare over all consumption streams, ${ }_{1} c \in C(b)$, while taking

14. The generalization of the analysis to the case where $g>0$ is straightforward but has a notational cost. Furthermore, in this case the result in propostion 2 on the monotonicity in time of the stream of brown capital holds only beyond period 1 , not between periods 0 and 1 .

15. The solution to this problem corresponds to the constrained optimal case of Rezai et al. (2012). Nordhaus (2008) is criticized by Rezai et al. (2012) for associating such a case with business as usual. 
the atmospheric externalities caused by the stream of brown capital, ${ }_{0} b \in K(b)$, as given. To facilitate the analysis of the appropriate problem in the model with atmospheric externalities, let the NPA welfare function $v_{\alpha}: \mathcal{K} \times \mathcal{K}$ be defined over capital streams as follows:

$$
v_{\alpha}\left({ }_{0} k,{ }_{0} b\right)=w_{\alpha}\left(\mathbf{u}\left(\mathbf{c}\left({ }_{0} k\right),{ }_{0} b\right)\right)=(1-\alpha) \sum_{t=0}^{\infty} \alpha^{t} a\left(b_{t}\right) u\left(k_{t}+f\left(k_{t}\right)-k_{t+1}\right),
$$

with $\alpha \in(0,1)$, where $k_{0}=b$ and $k_{t}$ is brown capital held by the representative dynasty for $t \in \mathbb{N}$. Note that the representative dynasty takes ${ }_{0} b$ as given when maximizing $v_{\alpha}\left({ }_{0} k,{ }_{0} b\right)$ over all ${ }_{0} k \in K(b)$. However, in equilibrium, ${ }_{0} k={ }_{0} b$, leading to the following definition: Say that ${ }_{0} b \in K(b)$ is an NPA equilibrium if

$$
v_{\alpha}\left({ }_{0} b,{ }_{0} b\right) \geq v_{\alpha}\left({ }_{0} \tilde{k},{ }_{0} b\right) \text { for all }{ }_{0} \tilde{k} \in K(b) .
$$

The following result is established in appendix B:

Proposition 2: Assume $b>0$ and $g=0$. Then there is a unique NPA equilibrium, $b^{*}(b)$, with associated NPA equilibrium consumption stream $c^{*}(b)=\mathbf{c}\left(k^{*}(b)\right)$. Furthermore, $b^{*}(b)$ is strictly monotone in time, with $\lim _{t \rightarrow \infty} b_{t}^{*}(b)=k_{\infty}(\alpha)$, and $c^{*}(b)$ is efficient, with $\lim _{t \rightarrow \infty} c_{t}^{*}(b)=f\left(k_{\infty}(\alpha)\right)$.

As for proposition 1 , we obtain the following corollary: ${ }^{16}$

Corollary 2: Assume $b>0$ and $g=0$. Let $\alpha^{*}=1 /\left(1+f^{\prime}(b)\right)$.

(a) If $\alpha \in\left(\alpha^{*}, 1\right)$, then the NPA equilibrium, $b^{*}(b)$, is increasing in time.

(b) If $\alpha=\alpha^{*}$, then the NPA equilibrium, $b^{*}(b)$, is constant in time.

(c) If $\alpha \in\left(0, \alpha^{*}\right)$, then the NPA equilibrium, $b^{*}(b)$, is decreasing in time.

Proposition 2 sets the stage for the following comparative statics result, which is also proven in appendix $\mathrm{B}^{17}$

16. While corollary 2 shows that the steady state stock of capital is not altered in presence of atmospheric externalities, the adjustment path to steady state changes. The fact that $b^{*}(b)$ increases in time in the case with $\alpha \in\left(\alpha^{*}, 1\right)$ makes investment by each dynasty less productive. So for a given stock of capital, the savings rate is smaller.

17. Rezai et al. (2012) highlight, in their parameter sensitivity analysis for their business-asusual case, that lowering the discount rate may lead to increased levels of atmospheric carbon and a larger capital stock. Theorem 2 formalizes such a result in the context of our model. Note that while the result is concerned with the infinite future, the negative effects start already in finite time. 
Theorem 2: Assume $b>0$ and $g=0$, and that all generations of each dynasty $i$ are nonpaternalistically altruistic according to (3) in the model with atmospheric externalities. Then long-term utility adjusted for atmospheric externalities,

$$
\lim _{t \rightarrow \infty} a\left(b_{t}^{*}(b)\right) u\left(c_{t}^{*}(b)\right)=a\left(k_{\infty}(\alpha)\right) u\left(f\left(k_{\infty}(\alpha)\right)\right),
$$

approaches 0 as $\alpha \rightarrow 1$.

This result brings forward in a stark manner how intergenerational altruism can be destructive, since caring more for future generations may seriously undermine future well-being. ${ }^{18}$ In the next section we show that the resulting outcomes cannot be endorsed by any of the criteria considered in appendix A, provided that altruism is sufficiently strong.

\section{EFFICIENT STREAMS}

It is a straightforward observation that the stream of adjusted utilities implemented by the dynasties through the NPA equilibrium is not efficient in the alternative model with atmospheric externalities if there is excessive altruism. In particular, if the altruism parameter $\alpha$ exceeds a critical level $\tilde{\alpha}$, defined by

$$
\tilde{\alpha}=\sup \left\{\alpha \in(0,1): a\left(k_{\infty}(\alpha)\right) u\left(f\left(k_{\infty}(\alpha)\right)\right) \geq u\left(f\left((1-\gamma) k_{\infty}(\alpha)\right)\right)\right\},
$$

then there exists a time $\tau \in \mathbb{N}$ such that adjusted utility will be increased for all $t>\tau$ by switching from brown to green capital for all periods beyond $\tau$, while keeping the stream unchanged for all $t \leq \tau$. This follows from the observation that the streams, $b_{t}^{*}(b)$ and $c_{t}^{*}(b)$, under decentralized decision making approaches $k_{\infty}(\alpha)$ and $f\left(k_{\infty}(\alpha)\right)$ as time goes to infinity. By the properties of $a$ and $k_{\infty}$, we have that the set in the brackets is nonempty and, furthermore, $\tilde{\alpha} \in(0,1)$.

Since efficiency is a necessary condition for Suppes-Sen maximality, it follows that the NPA equilibrium is not Suppes-Sen maximal if the altruism parameter $\alpha$ exceeds the critical level $\tilde{\alpha}$, defined by (6).

Although reported in the first part of theorem 3 below, this result stretches the plausibility of the assumptions of our model by letting the atmospheric externalities be removed by a sudden and total switch from brown to green capital. If, under additional structure on the $a$ function, it is possible to show that, for a sufficiently high altruism parameter $\alpha$, there exists a time $\tau$ such that $a\left(b_{\tau-1}^{*}\right) u\left(c_{\tau}^{*}\right)>a\left(k_{\infty}(\alpha)\right) u\left(f\left(k_{\infty}(\alpha)\right)\right)$, then simply stopping to accumulate brown capital at time $\tau$ would increase adjusted util-

18. As suggested in n. 6 and discussed in sec. 5, the problems discussed in this section cannot be solved by instead assuming altruism not only for the descendants in the same dynasty but also for the descendants of all dynasties. Imposing this assumption gives rise to another severe public good problem. 
ity for generation $\tau$ and all later generations, even if there is no switch to green capital. However, exploration of such possibilities is beyond the scope of this paper.

Note that in the model with atmospheric externalities efficient policies at a societal level require that the superior productiveness of brown capital is weighted against the atmospheric externalities that such capital leads to. In each period $t \in \mathbb{N}$, the mix of capital must be chosen so that the gain in production obtained by accumulating brown capital is weighted against the loss in adjusted utility, for given consumption, that such accumulation leads to.

To be specific, let $k$ be the total capital that is accumulated in period $t$, to be used in period $t+1$ and let $k^{\prime}$ be the total capital that is accumulated in period $t+1$, to be used in period $t+2$. Assume that $k^{\prime} \in[0, k+f(k))$ so that more than $k^{\prime}$ is attainable in period $t+1$ given $k$ in period $t$ with use of the more productive brown capital only. In period $t$, the asset management problem for given $k$ and $k^{\prime}$ is to split $k$ into brown capital, $\mu k$, and green capital, $(1-\mu) k$, so that

$$
a(\mu k) u\left(k+f(\mu k+(1-\gamma)(1-\mu) k)-k^{\prime}\right)
$$

is maximized over all $\mu \in\left[\mu\left(k, k^{\prime}\right), 1\right]$, where $\mu\left(k, k^{\prime}\right)<1$ is equal to 0 if $k^{\prime} \leq$ $k+f((1-\gamma) k)$ (so that $k^{\prime}$ is attainable also with the use of the less productive green capital only) and equal to the solution to the equation $k^{\prime}=k+f(\mu k+(1-\gamma)(1-$ $\mu) k$ ) otherwise. Obtaining such short-run efficiency is a necessary condition for an efficient stream of adjusted utility, because otherwise adjusted utility can be increased in period $t+1$ without being decreased in any other period.

Since the functions $a, u$, and $f$ are all continuous and $\left[\mu\left(k, k^{\prime}\right), 1\right]$ is a nonempty compact set, it follows that the expression is maximized for some $\mu$ and we may define:

$$
\hat{u}\left(k, k^{\prime}\right)=\max _{\mu \in\left[\mu\left(k, k^{\prime}\right), 1\right]} a(\mu k) u\left(k+f(\mu k+(1-\gamma)(1-\mu) k)-k^{\prime}\right) .
$$

It is sufficient for an NPA equilibrium stream not to be Suppes-Sen maximal that there exists some time $\tau$ where $a\left(b_{\tau}^{*}\right) u\left(c_{\tau+1}^{*}\right)<\hat{u}\left(b_{\tau}^{*}, b_{\tau+1}^{*}\right)$. It is difficult, however, to relate such violation of short-run efficiency to excessive altruism.

Making use of the above analysis of short-run efficiency, we now turn to the other normative criteria introduced in section 1 and presented in more detail in appendix A. Rather than analyzing directly streams that are optimal according to these criteria in the alternative model with atmospheric externalities, we establish the existence of an efficient maximin stream. As we will formalize in the discussion leading up to theorem 3, the welfare level associated with the efficient maximin stream is a lower bound for welfare according to the Calvo criterion, as well as the SDU and RDU criteria when varying the discount factor $\beta$ and the generalized utility function $v$. We use this in turn to show how excessive altruism leads to streams that are not optimal according to the Calvo, SDU, and RDU criteria. 
A maximin stream in the model with atmospheric externalities maximizes the infimum, taken over all time periods, of adjusted utility. If a stream with constant adjusted utility is efficient, so that adjusted utility cannot be increased in one period without being decreased in another period, then clearly the infimum of adjusted utility cannot be increased. Such an efficient stream with constant adjusted utility is an efficient maximin stream.

Let $b \geq 0$ and $g \geq 0$ be the initial stocks of brown and green capital, with $b+g>$ 0 . In appendix $\mathrm{C}$ we show that the unique maximin path is found by choosing total capital $\bar{k}$ from period 1 and on such that

$$
a(b) u(b+g+f(b+(1-\gamma) g)-\bar{k})=\hat{u}(\bar{k}, \bar{k}),
$$

leading to following constant stream of adjusted utilities:

$$
(a(b) u(b+g+f(b+(1-\gamma) g)-\bar{k}), \hat{u}(\bar{k}, \bar{k}), \ldots, \hat{u}(\bar{k}, \bar{k}), \ldots) .
$$

As stated in the following proposition this is indeed an efficient maximin stream:

Proposition 3: Assume $b \geq 0, g \geq 0$, and $b+g>0$. There is a unique maximin stream in the model with atmospheric externalities. This stream is efficient and has constant and positive adjusted utility.

Note that when this efficient maximin stream is followed, the NPA altruistic welfare is constant and equal to

$$
w_{\alpha}(\hat{u}(\bar{k}, \bar{k}), \hat{u}(\bar{k}, \bar{k}), \ldots)=(1-\alpha) \sum_{t=0}^{\infty} \alpha^{t} \hat{u}(\bar{k}, \bar{k})=\hat{u}(\bar{k}, \bar{k}) .
$$

Hence, when this efficient maximin stream is valued by the Calvo criterion, it yields $\hat{u}(\bar{k}, \bar{k})$, while it cannot exceed $\lim _{t \rightarrow \infty} a\left(b_{t}^{*}(b)\right) u\left(c_{t}^{*}(b)\right)=a\left(k_{\infty}(\alpha)\right) u\left(f\left(k_{\infty}(\alpha)\right)\right)$ in the case of decentralized decision making. Since the efficient maximin stream is feasible, the optimal stream according to the Calvo criterion gives rise to a welfare level at least as high as $\hat{u}(\bar{k}, \bar{k})$, implying that decentralized decision making cannot be Calvo optimal if $\lim _{t \rightarrow \infty} a\left(b_{t}^{*}(b)\right) u\left(c_{t}^{*}(b)\right)<\hat{u}(\bar{k}, \bar{k})$.

When this efficient regular stream is valued by the SDU and RDU criteria, it yields $v(\hat{u}(\bar{k}, \bar{k}))$ (where $v: \mathbb{R}_{+} \rightarrow \mathbb{R}$ is the increasing and continuous function that turns adjusted utility into generalized utility), while it cannot exceed $\lim _{t \rightarrow \infty} v\left(a\left(b_{t}^{*}(b)\right) u\left(c_{t}^{*}(b)\right)\right)=$ $v\left(a\left(k_{\infty}(\alpha)\right) u\left(f\left(k_{\infty}(\alpha)\right)\right)\right)$ in the case of decentralized decision making. Since the efficient maximin stream is feasible, the optimal streams according to the SDU and RDU criteria give rise to a welfare level at least as high as $v(\hat{u}(\bar{k}, \bar{k}))$ for any discount factor $\beta$ and any increasing and continuous generalized utility function $v$, implying that decentralized decision making cannot be SDU or RDU optimal if $\lim _{t \rightarrow \infty} a\left(b_{t}^{*}(b)\right) u\left(c_{t}^{*}(b)\right)<\hat{u}(\bar{k}, \bar{k})$. 
Hence, it follows from theorem 2 that the outcome of decentralized decision making cannot be endorsed by any of these criteria, if the altruism parameter exceeds a critical level $\bar{\alpha}$, defined by

$$
\bar{\alpha}=\sup \left\{\alpha \in(0,1): a\left(k_{\infty}(\alpha)\right) u\left(f\left(k_{\infty}(\alpha)\right)\right) \geq \hat{u}(\bar{k}, \bar{k})\right\},
$$

if the set in the brackets is nonempty and $\bar{\alpha}=0$ otherwise. It follows from the properties of $a$ and $k_{\infty}$ that $\bar{\alpha} \in[0,1)$.

Theorem 3: Assume $b>0$ and $g=0$, and that all generations of each dynasty $i$ are nonpaternalistically altruistic according to (3) in the model with atmospheric externalities. Then the implemented pair of capital streams, $b^{*}(b)$ and ${ }_{0} g=(0,0, \ldots, 0, \ldots)$, with associated consumption stream, $c^{*}(b)=c\left(b^{*}(b)\right)$, is

- not Suppes-Sen maximal

if $\alpha>\tilde{\alpha}$, so that long-term utility, $\lim _{t \rightarrow \infty} a\left(b_{t}^{*}(b)\right) u\left(c_{t}^{*}(b)\right)$, adjusted for atmospheric externalities, would be increased by shifting from brown to green capital, and

- not Calvo optimal, and

- not SDU optimal nor RDU optimal for any $\beta \in(0,1)$ and any increasing and continuous $v$ function,

if $\alpha>\bar{\alpha}$, so that long-term utility, $\lim _{t \rightarrow \infty} a\left(b_{t}^{*}(b)\right) u\left(c_{t}^{*}(b)\right)$, adjusted for atmospheric externalities, is smaller than the maximin level, $\hat{u}(\bar{k}, \bar{k})$.

\section{COLLECTIVE ACTION}

A feasible policy is to require the dynasties to accumulate green capital only. This can be done by direct command or by imposing a prohibitive tax on brown capital. If such policies are enforced, then the dynasties will accumulate green capital only and the atmospheric externalities will be avoided. Under the assumption that $b=0$ and $g>0$, so that also in period 0 there is a positive stock of green capital only, and replacing $f(g)$ with $\tilde{f}(g)=f((1-\gamma) g)$ in the original Ramsey model of section 1 , the analysis becomes straightforward.

Denote by

$$
G(g)=\left\{0 g: g_{0}=g \text { and } 0 \leq g_{t} \leq g_{t-1}+\tilde{f}\left(g_{t-1}\right) \text { for all } t \in \mathbb{N}\right\}
$$

the set of feasible green capital streams subject to ${ }_{0} b=(0,0, \ldots, 0, \ldots)$, as a function of the initial stock of green capital $g$, and define

$$
\tilde{\mathbf{c}}(0 g)=\left(g_{0}+\tilde{f}\left(g_{0}\right)-g_{1}, g_{1}+\tilde{f}\left(g_{1}\right)-g_{2}, \ldots, g_{t-1}+\tilde{f}\left(g_{k-1}\right)-g_{t}, \ldots\right)
$$

as the constrained consumption stream that is associated with ${ }_{0} g$.

Say that ${ }_{0} g \in G(g)$ is a constrained NPA optimum if

$$
v_{\alpha}\left({ }_{0} g\right) \geq v_{\alpha}\left({ }_{0} \tilde{g}\right) \text { for all } 0 \tilde{g} \in G(g) .
$$


Before characterizing the constrained optimum, we need to redefine the modified golden rule. Define $g_{\infty}:(0,1) \rightarrow \mathbb{R}_{+}$by, for all $\alpha \in(0,1)$,

$$
\alpha\left(1+(1-\gamma) \tilde{f}^{\prime}\left(g_{\infty}(\alpha)\right)\right)=1 .
$$

It follows from the properties of $\tilde{f}$ that $g_{\infty}$ is well defined, continuous, and increasing, with $\lim _{\alpha \rightarrow 0} g_{\infty}(\alpha)=0$ and $\lim _{\alpha \rightarrow 1} g_{\infty}(\alpha)=\infty$. For given $\alpha \in(0,1), g_{\infty}(\alpha)$ is the green capital stock corresponding to the modified golden rule.

As in section 1, the analysis of Beals and Koopmans (1969) leads to the following result:

Proposition 4: Assume $b=0$ and $g>0$ and impose the constraint that $b_{t}=0$ for all $t \in \mathbb{N}$. Then there is a unique constrained NPA optimum, $\hat{g}(g)$, with associated constrained NPA optimal consumption stream $\hat{c}(g)=\tilde{\mathbf{c}}(\hat{g}(g))$. Furthermore, $\hat{g}(g)$ is strictly monotone in time, with $\lim _{t \rightarrow \infty} \hat{g}_{t}(g)=g_{\infty}(\alpha)$, and $\hat{c}(g)$ is constrained efficient, with $\lim _{t \rightarrow \infty} \hat{c}_{t}(g)=\tilde{f}\left(g_{\infty}(\alpha)\right)$.

Furthermore, the constrained NPA optimum, $\hat{g}(g)$, is increasing in time if $\alpha \in$ $\left(\alpha^{*}, 1\right)$, constant in time if $\alpha=\alpha^{*}$, and decreasing in time if $\alpha \in\left(0, \alpha^{*}\right)$, where $\alpha^{*}=1 /\left(1+(1-\gamma) \tilde{f}^{\prime}(g)\right)$.

The policy of accumulating green capital only need not be efficient in terms of adjusted utility if short-run efficiency (as defined by problem [7]) requires a mixture of brown and green capital with a positive amount of brown capital. This will be the case if $\gamma$ is large, so that brown capital is much more productive than green capital, and $a$ is only moderately decreasing, so that the effects of the atmospheric externalities are small.

In the opposite case, where $\gamma$ is small and a decreases steeply with the amount of brown capital, then the efficiently loss of such a policy may be small or even nonexistent. Indeed, the following result, proven in appendix $\mathrm{D}$, shows under what condition the efficiency loss in terms of adjusted utility is nonexistent in the special case where the function capturing the effects of the atmospheric externalities is given by $a(\mu k)=$ $e^{-\rho \mu k}$, with $\rho>0$. The theorem demonstrates that, for any $\rho>0$, there is a $\gamma>0$ small enough to ensure that the efficiency loss in terms of adjusted utility associated with accumulating green capital only is zero.

Theorem 4: Assume $b=0$ and $g>0$. Also, assume $a(\mu k)=e^{-\rho \mu k}$, with $\rho>0$. Then the constrained NPA optimum, $\hat{g}(g)$, with associated constrained NPA optimal consumption stream $\hat{c}(g)=\tilde{\boldsymbol{c}}(\hat{g}(g))$, is short-run efficient in all periods if and only if $\gamma \in(0, \gamma(\rho)]$, where $\gamma(\rho)>0$ is an increasing function of $\rho$, with $\lim _{\rho \rightarrow 0} \gamma(\rho)=0$.

Furthermore, with a small $\gamma$, accumulating green capital only will lead to behavior and outcomes that are close to those obtained in the ordinary Ramsey model. As we 
have shown in sections 2 and 3, the behavior and outcomes will be very different and adverse without such collective action, even in the case where $\gamma$ is small.

\section{DISCUSSION}

As stated in the introduction, the purpose of this paper is to construct a simple model where increased intergenerational altruism has serious counterproductive consequences in a setting with atmospheric externalities. We now discuss whether the main results are robust to relaxing some core assumptions.

\section{Caring for the Descendants of All Dynasties}

The results of this paper have been derived under the assumption that present members of dynasties care only for their own descendants. Suppose, instead, that present members of dynasties care not only for the descendants in the same dynasty, but for all descendants. For simplicity, let dynasties have the same utility function $u$ and that the profile of initial ownership to capital is uniform, so that $\mathbf{k}_{0}(i)=k$ for all $i \in[0,1]$. Define $\mathbf{u}_{t}(i)=u\left(\mathbf{c}_{t}(i)\right)$. Assume now that the altruistic welfare of the representative dynasty in period $t$ depends linearly on the utility of the current generation of the dynasty and the aggregate altruistic welfare of the next generation of all dynasties:

$$
\mathbf{w}_{t}(i)=(1-\alpha) \mathbf{u}_{t}(i)+\alpha \int_{0}^{1} \mathbf{w}_{t+1}(j) d j,
$$

where $\alpha \in(0,1)$.

Under centralized decision making aggregate welfare, $\int_{0}^{1} \mathbf{w}_{1}(j) d j$, is maximized subject to equation (10) recursively. Because the maximand of this problem is in the aggregate identical to the maximand for the problem of equation (3), the altruistic welfare of each dynasty will be identical. In particular, the implemented stream will be identical to the optimum of the ordinary Ramsey model of section 1.

Note, however, that the member of the representative dynasty in period $t$ seeks to maximize equation (10) with respect to own utility. As the second term of the righthand side of this expression cannot be influenced by the investment behavior of the representative dynasty, no assets will be accumulated. The stream of the ordinary Ramsey model can therefore not be implemented through decentralized decision making of this form. In consequence, problems encountered in sections 2 and 3 cannot be solved by assuming that the present members of dynasties care for the descendants of all dynasties. Imposing this assumption gives rise to another and more serious public good problem, namely, that present members want to free-ride in providing for future generations.

\section{The Weight Assigned to Each Dynasty}

As we are interested in investigating an economy divided into dynasties that are small compared to the aggregate economy, the assumption that each dynasty is of zero weight is an appropriate representation. The word "dynasty" is meant to capture fam- 
ilies or tribes that, under the assumptions of sections 2 and 3, live in a setting where there is no climate policy at the level of countries nor at the level of coalitions of countries. Thus, no single dynasty is able to influence the aggregate stock of brown capital that adjusts its utility.

\section{Learning by Doing in the Accumulation and Use of Green Capital}

By assuming that $\gamma$, the measure to what extent green capital is less productive than brown capital, is constant in the model with atmospheric externalities analyzed in section 2, we abstract from directed technological progress. Suppose, instead, that technological progress is a learning process in the accumulation and use of green capital, with the technology of brown capital being considered mature. So assume that $\gamma$, rather than being a fixed parameter, is a function of prior accumulation and use of green capital, but with initial value between 0 and 1 . We have two cases to consider, depending on whether learning is private or publicly shared.

With private learning, $\gamma$ is an individual parameter for each dynasty, and it depends on the dynasty's prior accumulation and use of green capital. It is clear that if $\gamma$ remains nonnegative, independently of prior accumulation and use, then it will pay the dynasty to use brown capital only. Hence, learning by doing will never get started.

With publicly shared learning, $\gamma$ is a common parameter that depends on the aggregate prior accumulation and use of green capital. In this case, learning is a public good to which no dynasty will contribute. This holds even if green capital might obtain a cost advantage relative to brown capital (corresponding to a negative $\gamma$ ) after sufficient prior accumulation and use. The reason is that, initially, investing in brown rather than green capital will relax the representative dynasty's budget constraint, while its investment behavior will not influence the aggregate learning process. Hence, also in this case, learning by doing will never get started, and this even if $\gamma$ might eventually become negative.

The conclusion is that our results are robust to allowing for learning by doing in the accumulation and use of green capital, except in the special case where learning is private and $\gamma$ eventually becomes negative, so that green capital ends up with having a cost advantage in comparison to brown capital.

\section{Substitution Possibilities between Brown and Green Capital}

We make the simplifying assumption of perfect substitutability between brown and green capital. The following argument implies that this assumption comes without much loss of generality: Interpret brown capital as the capital mix chosen by each dynasty in absence of collective climate policy, leading to some atmospheric externalities. Interpret green capital as a capital mix that is cost efficient when constrained to having zero atmospheric externalities. In a potential real-world setting of an efficient collective climate policy, either a mixture of brown and green capital or green capital only should be chosen, which is the same conclusion that we obtain within our simplified modeling. 


\section{CONCLUSION}

In this paper we have shown how intergenerational altruism may seriously undermine long-term well-being in a setting that is designed to model the intergenerational game that will be played by dynasties trying to get ahead in a world threatened by climate change. The main insight is that stronger concern for future generations need not be an effective substitute for collective climate action. If the helping hand that present members of each dynasty lend to its future members accelerates climate change, then increased uncoordinated transfers toward the future might aggravate the conditions under which future generations will live.

\section{APPENDIX A}

Definitions of Suppes-Sen Maximality, Calvo Optimality, TDU Optimality, SDU Optimality, and RDU Optimality

Let $U$ be a set of feasible utility streams for a given initial condition ( $k$ in the model of sec. 1 and $(b, g)$ in the model of sec. 2). That is, the utility stream ${ }_{1} u=\left(u_{1}, u_{2}, \ldots\right)$ is feasible if and only if ${ }_{1} u \in U$. Let $\mathcal{U}$ be the union of $U$ when varying the initial conditions in both models.

Suppes-Sen maximality (see Asheim et al. [2001] for a presentation and analysis of this criterion).

Say that the utility stream ${ }_{1} u \in U$ is Suppes-Sen maximal given the set of feasible utility streams $U$ if there do not exist ${ }_{1} \tilde{u} \in U$ and a finite permutation ${ }_{1} u^{\prime}$ of ${ }_{1} u$ such that ${ }_{1} \tilde{u}>{ }_{1} u^{\prime}$.

The Calvo criterion (see Calvo [1978] for a presentation and analysis of this criterion) evaluates streams according to the infimum of the nonpaternalistic altruistic welfare. Hence, the Calvo welfare function is defined on $\mathcal{U}$ as follows:

$$
\inf _{t \geq 1} w_{\alpha}\left({ }_{t} u\right) .
$$

Say that ${ }_{1} u \in U$ is a Calvo optimum if

$$
\inf _{t \geq 1} w_{\alpha}\left({ }_{t} u\right) \geq \inf _{t \geq 1} w_{\alpha}\left({ }_{t} \tilde{u}\right) \text { for all }{ }_{1} \tilde{u} \in U .
$$

Let $v: \mathbb{R}_{+} \rightarrow \mathbb{R}$ be a continuous and increasing function that maps utility into generalized utility.

Time-discounted generalized utilitarianism (TDU). Define the TDU welfare function $W_{\beta}^{T}: \mathcal{U} \rightarrow \mathbb{R}$ for the discount factor $\beta \in(0,1)$ as follows:

$$
W_{\beta}^{T}\left({ }_{1} u\right)=(1-\beta) \sum_{t=0}^{\infty} \beta^{t} v\left(u_{t+1}\right) .
$$

Say that ${ }_{1} u \in U$ is a time-discounted generalized utilitarian (TDU) optimum given the set of feasible utility streams $U$ if 


$$
W_{\beta}^{T}\left({ }_{1} u\right) \geq W_{\beta}^{T}\left({ }_{1} \tilde{u}\right) \text { for all }{ }_{1} \tilde{u} \in U .
$$

Sustainable discounted generalized utilitarianism (SDU) (see Asheim and Mitra [2010] for a presentation and analysis of this criterion, including an axiomatization). Under SDU, the future is discounted if and only if the future is better off than the present. Define the SDU welfare function $W_{\beta}^{S}: \mathcal{U} \rightarrow \mathbb{R}$ for $\beta \in(0,1)$ as follows: $W_{\beta}^{S}\left({ }_{1} u\right)=$ $\lim _{\tau \rightarrow \infty} z(1, \tau)$, where $z(1, \tau)$ is constructed as follows:

$$
\begin{aligned}
& z(\tau, \tau)=W_{\beta}^{T}\left({ }_{\tau} u\right) \\
& z(\tau-1, \tau)=\min \left\{(1-\beta) v\left(u_{\tau-1}\right)+\beta z(\tau, \tau), z(\tau, \tau)\right\} \\
& \ldots \\
& z(1, \tau)=\min \left\{(1-\beta) v\left(u_{1}\right)+\beta z(2, \tau), z(2, \tau)\right\} .
\end{aligned}
$$

Say that ${ }_{1} u \in U$ is a sustainable generalized discounted utilitarian (SDU) optimum given the set of feasible utility streams $U$ if ${ }^{19}$

$$
W_{\beta}^{S}\left({ }_{1} u\right) \geq W_{\beta}^{S}\left({ }_{1} \tilde{u}\right) \text { for all }{ }_{1} \tilde{u} \in U .
$$

Rank-discounted generalized utilitarianism (RDU) (see Zuber and Asheim [2012] for a presentation and analysis of this criterion, including an axiomatization). Under $\mathrm{RDU}$, streams are first reordered into a nondecreasing stream, so that discounting becomes according to rank, not according to time. The definition takes into account that streams like $(1,0,0,0, \ldots)$, with elements of infinite rank, cannot be reordered into a nondecreasing stream. Therefore, let $\ell\left({ }_{1} u\right)$ denote lim inf of ${ }_{1} u$ if it exists $\left(\operatorname{set} \ell\left({ }_{1} u\right)=\right.$ $\infty$ otherwise), and let $L\left({ }_{1} u\right):=\left\{t \in \mathbb{N} \mid u_{t}<\ell\left({ }_{1} u\right)\right\}$. If $\left|L\left({ }_{1} u\right)\right|=\infty$, then let ${ }_{[1]} u=$ $\left(u_{[1]}, u_{[2]}, \ldots\right)$ be a nondecreasing reordering of all elements $u_{t}$ with $t \in L\left({ }_{1} u\right)$ (so that $u_{[r]} \leq u_{[r+1]}$ for all ranks $\left.r \in \mathbb{N}\right)$. If $\left|L\left({ }_{1} u\right)\right|<\infty$, then let $\left(u_{[1]}, u_{[2]}, \ldots, u_{\left[\left|L L_{1} u\right|\right]}\right)$ be a nondecreasing reordering of all elements $u_{t}$ with $t \in L\left({ }_{1} u\right)$ (so that $u_{[r]} \leq u_{[r+1]}$ for all ranks $\left.r \in\left\{1, \ldots,\left|L\left({ }_{1} u\right)\right|\right\}\right)$, and set $u_{[r]}=\ell\left({ }_{1} u\right)$ for all $r>\left|L\left({ }_{1} u\right)\right|$.

Define the RDU welfare function $W_{\beta}^{R}: \mathcal{U} \rightarrow \mathbb{R}$ for $\beta \in(0,1)$ as follows:

$$
W_{\beta}^{R}\left({ }_{1} u\right)=W_{\beta}^{T}\left({ }_{[1]} u\right) .
$$

Say that ${ }_{1} u \in U$ is a rank-discounted generalized utilitarian (RDU) optimum given the set of feasible utility streams $U$ if

$$
W_{\beta}^{R}\left({ }_{1} u\right) \geq W_{\beta}^{R}\left({ }_{1} \tilde{u}\right) \text { for all }{ }_{1} \tilde{u} \in U .
$$

19. Asheim and Mitra (2010, sec. 2) use the construction presented here to establish the existence of an SDU welfare function, while using their requirements (W.1)-(W.4) as the primitive definition. 
It follows from the assumptions made in sections 1 and 2 that the generalized utilitarian welfare functions $W_{\beta}^{T}, W_{\beta}^{S}$, and $W_{\beta}^{R}$ are well defined on $\mathcal{U}$ if the function $v$ that turns utility into generalized utility is (weakly) concave.

\section{APPENDIX B}

\section{Proof of Proposition 2}

Throughout this proof, we assume that ${ }_{0} g=0$ so that the set of brown capital streams for the representative dynasty is given by $K(b)$. In particular, the transformation between the stock of brown capital at time $t-1$, denoted $k_{t-1}$, and the stock of brown capital at time $t$, denoted $k_{t}$, provided that $c_{t}$ is consumed at time $t$, is given by equation (1).

A necessary condition for the maximization by ${ }_{0} k \in K(b)$ of $v_{\alpha}\left({ }_{0} \tilde{k},{ }_{0} b\right)$ over all ${ }_{0} \tilde{k} \in K(b)$ is short-run optimality in the following sense: for all $t \in \mathbb{N}, k_{t-1}>0$, and $k_{t+1} \in\left[0, k_{t-1}+f\left(k_{t-1}\right)+f\left(k_{t-1}+f\left(k_{t-1}\right)\right)\right], k_{t}$ maximizes

$$
a\left(b_{t-1}\right) u\left(k_{t-1}+f\left(k_{t-1}\right)-\tilde{k}_{t}\right)+\alpha a\left(b_{t}\right) u\left(\tilde{k}_{t}+f\left(\tilde{k}_{t}\right)-k_{t+1}\right)
$$

over all $\tilde{k}_{t} \leq k_{t-1}+f\left(k_{t-1}\right)$, satisfying $\tilde{k}_{t}+f\left(\tilde{k}_{t}\right) \geq k_{t+1}$. Because otherwise, a reallocation of consumption between periods $t$ and $t+1$ could have increased $v_{\alpha}\left({ }_{0} k,{ }_{0} b\right)$ without affecting consumption in any other period. This yields the first-order condition

$$
a\left(b_{t-1}\right) u^{\prime}\left(c_{t}\right)=\alpha a\left(b_{t}\right) u^{\prime}\left(c_{t+1}\right)\left(1+f^{\prime}\left(k_{t}\right)\right),
$$

where $c_{t}=k_{t-1}+f\left(k_{t-1}\right)-k_{t}>0$ and $c_{t+1}=k_{t}+f\left(k_{t}\right)-k_{t+1}>0$ by the properties of $u$. Equation (B1) is the key to proving the following useful result.

Lemma 1: Assume that ${ }_{0} k \in K(b)$ maximizes $v_{\alpha}\left({ }_{0} \tilde{k},{ }_{0} b\right)$ over all ${ }_{0} \tilde{k} \in K(b)$ and ${ }_{0} k^{\prime} \in K\left(b^{\prime}\right)$ maximizes $v_{\alpha}\left({ }_{0} \tilde{k},{ }_{0} b^{\prime}\right)$ over all ${ }_{0} \tilde{k} \in K\left(b^{\prime}\right)$. If, for some $t \in \mathbb{N}, k_{t}=$ $b_{t} \leq k_{t}^{\prime}=b_{t}^{\prime}$ and $a\left(b_{t-1}\right) u^{\prime}\left(c_{t}\right)<a\left(b_{t-1}^{\prime}\right) u^{\prime}\left(c_{t}^{\prime}\right)$, then $a\left(b_{t}\right) u^{\prime}\left(c_{t+1}\right)<a\left(b_{t}^{\prime}\right) u^{\prime}\left(c_{t+1}^{\prime}\right)$, $c_{t+1}>c_{t+1}^{\prime}$, and $k_{t+1}<k_{t+1}^{\prime}$.

Proof: It follows from (B1), $k_{t}=b_{t} \leq k_{t}^{\prime}=b_{t}^{\prime}, a\left(b_{t-1}\right) u^{\prime}\left(c_{t}\right)<a\left(b_{t-1}^{\prime}\right) u^{\prime}\left(c_{t}^{\prime}\right)$, the monotonicity of $a$ and the strict concavity of $f$ that

$$
\begin{aligned}
& a\left(b_{t}\right) u^{\prime}\left(c_{t+1}\right)\left(1+f^{\prime}\left(k_{t}\right)\right)<a\left(b_{t}^{\prime}\right) u^{\prime}\left(c_{t+1}^{\prime}\right)\left(1+f^{\prime}\left(k_{t}^{\prime}\right)\right) \\
\leq & a\left(b_{t}^{\prime}\right) u^{\prime}\left(c_{t+1}^{\prime}\right)\left(1+f^{\prime}\left(k_{t}\right)\right) \leq a\left(b_{t}\right) u^{\prime}\left(c_{t+1}^{\prime}\right)\left(1+f^{\prime}\left(k_{t}\right)\right) .
\end{aligned}
$$

Hence, $a\left(b_{t}\right) u^{\prime}\left(c_{t+1}\right)<a\left(b_{t}^{\prime}\right) u^{\prime}\left(c_{t+1}^{\prime}\right)$ and $u^{\prime}\left(c_{t+1}\right)<u^{\prime}\left(c_{t+1}^{\prime}\right)$. By the strict concavity of $u$, we have that $c_{t+1}>c_{t+1}^{\prime}$. Since $k_{t} \leq k_{t}^{\prime}$, it follows from equation (1) and the monotonicity of $f$ that $c_{t+1}+k_{t+1}=k_{t}+f\left(k_{t}\right) \leq k_{t}^{\prime}+f\left(k_{t}^{\prime}\right)=c_{t+1}^{\prime}+k_{t+1}^{\prime}$, which combined with $c_{t+1}>c_{t+1}^{\prime}$ implies that $k_{t+1}<k_{t+1}^{\prime}$. QED 
The proof of proposition 2 modifies Beals and Koopmans's (1969) proof of their theorem 1 and will be divided into nine steps.

Step 1: For every $b \in \mathbb{R}_{+}$, the set of streams $K(b)$ is compact. Set $k_{0}^{*}=b$ and define ${ }_{1} k^{*}$ recursively by $k_{t}^{*}=k_{t-1}^{*}+f\left(k_{t-1}^{*}\right)$ for $t \in \mathbb{N}$. So ${ }_{0} k^{*}$ is the stream with no consumption. $K(b)$ is contained in the product of the closed intervals $\left[0, k_{t}^{*}\right]$. The latter set is compact and $K(b)$ is a closed subset.

Step 2: For given ${ }_{0} b, v_{\alpha}\left({ }_{0} k,{ }_{0} b\right)$ is continuous and strictly quasi-concave as a function of ${ }_{0} k$. This follows from the properties of $u$.

Step 3: For given ${ }_{0} b$, there exists a unique NPA optimal stream $\hat{k}\left(b,{ }_{0} b\right)$ and the associated consumption stream $\hat{c}\left(b,{ }_{0} b\right)=\boldsymbol{c}\left(\hat{k}\left(b,{ }_{0} b\right)\right)$ is efficient. Existence follows from Weierstrass's extreme value theorem and uniqueness from the strict quasi-concavity of $v_{\alpha}\left({ }_{0} k,{ }_{0} b\right)$ as a function of ${ }_{0} k$. The optimality of $\hat{k}\left(b,{ }_{0} b\right)$ implies that $\hat{c}\left(b,{ }_{0} b\right)$ is efficient.

Step 4: If $b>0$, then $\hat{k}\left(b,{ }_{0} b\right) \gg 0$ and $\hat{c}\left(b,{ }_{0} b\right) \gg 0$. If $k_{t-1}>0$, then short optimality (cf. [B1]) implies that $c_{t}=k_{t-1}+f\left(k_{t-1}\right)-k_{t}>0, c_{t+1}=k_{t}+f\left(k_{t}\right)-k_{t+1}>0$, and $k_{t}>0$. Since $k_{0}=b>0$, the result follows by induction.

Step $5: \hat{k}\left(b,{ }_{0} b\right)$ is continuous in ${ }_{0} b$. Write $\hat{v}_{\alpha}\left(b,{ }_{0} b\right)=v_{\alpha}\left(\hat{k}\left(b,{ }_{0} b\right),{ }_{0} b\right)$.

We first establish that $\hat{v}_{\alpha}\left(b,{ }_{0} b\right)$ is strictly decreasing in $b_{t}$ and continuous from the right in $b_{t}$. Let $\tilde{b}>b_{t}$ and let ${ }_{0} b^{\prime}$ be given by $b_{t}^{\prime}=\tilde{b}$ and $b_{\tau}^{\prime}=b_{\tau}$ for $\tau \neq t$. Then

$$
\hat{v}_{\alpha}\left(b,{ }_{0} b\right) \geq v_{\alpha}\left(\hat{k}\left(b,{ }_{0} b^{\prime}\right),{ }_{0} b\right)>v_{\alpha}\left(\hat{k}\left(b,{ }_{0} b^{\prime}\right),{ }_{0} b^{\prime}\right)=\hat{v}_{\alpha}\left(b,{ }_{0} b^{\prime}\right)
$$

since going from ${ }_{0} b$ to ${ }_{0} b^{\prime}$ does not influence $K(b)$ but decreases adjusted utility derived from $\hat{k}\left(b,{ }_{0} b^{\prime}\right)$ at time $t+1$ from $a\left(b_{t}\right) u^{\prime}\left(\hat{c}_{t+1}\left(b,{ }_{0} b^{\prime}\right)\right)$ to $a\left(b_{t}^{\prime}\right) u^{\prime}\left(\hat{c}_{t+1}\left(b,{ }_{0} b^{\prime}\right)\right)$. Moreover,

$$
\hat{v}_{\alpha}\left(b,{ }_{0} b\right)>\hat{v}_{\alpha}\left(b,{ }_{0} b^{\prime}\right) \geq v_{\alpha}\left(\hat{k}\left(b,{ }_{0} b\right),{ }_{0} b^{\prime}\right),
$$

using the property that $\hat{v}_{\alpha}\left(b,{ }_{0} b\right)$ is strictly decreasing in $b_{t}$. Continuity from the right follows since $v_{\alpha}$ is continuous in $b_{t}$.

Suppose ${ }_{0} b^{(n)} \rightarrow{ }_{0} b$. Since $K(b)$ is compact, $\hat{k}\left(b,{ }_{0} b^{(n)}\right)$ has at least one convergent subsequence. It suffices to show that any convergent subsequence must converge to $\hat{k}\left(b,{ }_{0} b\right)$. Dropping terms and renumbering, we may assume that $\hat{k}\left(b,{ }_{0} b^{(n)}\right)$ itself converges to ${ }_{0} k$. By continuity of $v_{\alpha}$, we have that

$$
v_{\alpha}\left({ }_{0} k,{ }_{0} b\right)=\lim _{n \rightarrow \infty} v_{\alpha}\left(\hat{k}\left(b,{ }_{0} b^{(n)}\right),{ }_{0} b^{(n)}\right)=\lim _{n \rightarrow \infty} \hat{v}_{\alpha}\left(b,{ }_{0} b^{(n)}\right) \geq \hat{v}_{\alpha}\left(b,{ }_{0} b\right) \geq v_{\alpha}\left({ }_{0} k,{ }_{0} b\right),
$$

using the fact that $\hat{v}_{\alpha}\left(b,{ }_{0} b\right)$ is strictly decreasing in $b_{t}$ and continuous from the right in $b_{t^{*}}$ Therefore, $v_{\alpha}\left({ }_{0} k,{ }_{0} b\right)=\hat{v}_{\alpha}\left(b,{ }_{0} b\right)=v_{\alpha}\left(\hat{k}\left(b,{ }_{0} b\right),{ }_{0} b\right)$, and by the uniqueness of $\hat{k}\left(b,{ }_{0} b\right),{ }_{0} k=\hat{k}\left(b,{ }_{0} b\right)$. This proves that $\hat{k}\left(b,{ }_{0} b\right)$ is continuous in ${ }_{0} b$.

Step 6: For given 0 , there exists a unique NPA equilibrium stream $b^{*}(b)$ where associated consumption stream $c^{*}(b)=\mathbf{c}\left(b^{*}(b)\right)$ is efficient. By steps 1 and 5 , Brouwer's fixed point theorem implies that there exists ${ }_{0} \hat{b} \in K(b)$ such that ${ }_{0} \hat{b}=\hat{k}\left(b,{ }_{0} \hat{b}\right)$. 
To show that ${ }_{0} \hat{b}$ is unique, let ${ }_{0} \hat{b}, \hat{b}_{0}^{\prime} \in K(b)$ satisfy ${ }_{0} \hat{b}=\hat{k}\left(b,{ }_{0} \hat{b}\right)$ and ${ }_{0} \hat{b}^{\prime}=\hat{k}\left(b,{ }_{0} \hat{b}^{\prime}\right)$. Note that $\hat{b}_{0}=\hat{b}_{0}^{\prime}=b$. Suppose that there is a first time $t \in \mathbb{N}$ such that $\hat{b}_{t}<\hat{b}_{t^{\prime}}^{\prime}$ Then, by equation $(1), \hat{c}_{t}\left(b,{ }_{0} \hat{b}\right)>\hat{c}_{t}\left(b,{ }_{0} \hat{b}^{\prime}\right)$ and $a\left(\hat{b}_{t-1}\right) u^{\prime}\left(\hat{c}_{t}\left(b,{ }_{0} \hat{b}\right)\right)<a\left(\hat{b}_{t-1}^{\prime}\right) u^{\prime}\left(\hat{c}_{t}\left(b, \hat{b}_{0}\right)\right)$, keeping in mind that $\hat{b}_{t-1}=\hat{b}_{t-1}^{\prime}$. By repeated use of lemma $1, \hat{c}_{\tau}\left(b,{ }_{0} \hat{b}\right)>\hat{c}_{\tau}\left(b,{ }_{0} \hat{b}^{\prime}\right)$ for all $\tau \geq t$. Since $\hat{c}_{\tau}\left(b,{ }_{0} \hat{b}\right)=\hat{c}_{\tau}\left(b,{ }_{0} \hat{b}^{\prime}\right)$ for all $\tau=1, \ldots, t-1$ if $t>1$, it follows that $\hat{c}_{t}\left(b, \hat{b}^{\prime}\right)$ is inefficient. By step 3 , this leads to a contradiction.

The efficiency of $c^{*}(b)$ follows from step 3 .

Step 7: If $b>b^{\prime}>0$, then $b^{*}(b) \gg b^{*}\left(b^{\prime}\right)$. Let $b>b^{\prime}>0$, and suppose that $b_{1}^{*}(b) \leq b_{1}^{*}\left(b^{\prime}\right)$. Then, by equation (1) and $b_{0}^{*}(b)=b>b^{\prime}=b_{0}^{*}\left(b^{\prime}\right)$, it follows that $c_{1}^{*}(b)>c_{1}^{*}\left(b^{\prime}\right)$ and $a\left(b_{0}^{*}(b)\right) u^{\prime}\left(c_{1}^{*}(b)\right)<a\left(b_{0}^{*}\left(b^{\prime}\right)\right) u^{\prime}\left(c_{1}^{*}\left(b^{\prime}\right)\right)$ by the properties of $a$ and $u$. By repeated use of lemma $1, c_{t}^{*}(b)>c_{t}^{*}\left(b^{\prime}\right)$ and $b_{t}^{*}(b)<b_{t}^{*}\left(b^{\prime}\right)$ for all $t \geq 2$.

If $b_{1}^{*}(b)=b_{1}^{*}\left(b^{\prime}\right)=b_{1}$, then this would contradict that $b^{*}\left(b_{1}\right)$ is unique.

If $b_{1}^{*}(b)<b_{1}^{*}\left(b^{\prime}\right)$, then the conclusion that $c^{*}\left(b_{1}^{*}(b)\right)$ strictly dominates $c^{*}\left(b_{1}^{*}\left(b^{\prime}\right)\right)$ contradicts the efficiency of $c^{*}\left(b_{1}^{*}\left(b^{\prime}\right)\right)$.

Therefore, $b_{1}^{*}(b)>b_{1}^{*}\left(b^{\prime}\right)$. Repeating the argument implies that $b^{*}(b) \gg b^{*}\left(b^{\prime}\right)$.

Step 8: $b^{*}(b)$ is strictly monotone in time. Assume $b=b_{0}^{*}(b)<b_{1}^{*}(b)$. Then, by step $7, b_{t-1}^{*}(b)<b_{t}^{*}(b)$ for all $t \in \mathbb{N}$. Likewise for $b=b_{0}^{*}(b)=b_{1}^{*}(b)$ and $b=$ $b_{0}^{*}(b)>b_{1}^{*}(b)$.

Step 9: $b^{*}(b)$ converges to $k_{\infty}(\alpha)$. By step $8, b^{*}(b)$ is strictly monotone in time and there are three possible limits for $b_{t}^{*}(b)$ as $t \rightarrow \infty$ :

(i) $b^{*}(b)$ is decreasing with $\lim _{t \rightarrow \infty} b_{t}^{*}(b)=0$,

(ii) $b^{*}(b)$ is increasing with $\lim _{t \rightarrow \infty} b_{t}^{*}(b)=\infty$,

(iii) $b^{*}(b)$ is strictly monotone with $\lim _{t \rightarrow \infty} b_{t}^{*}(b) \in(0, \infty)$.

In case (i), the properties of $f$ imply that there is $t \in \mathbb{N}$ such that $\alpha(1+$ $\left.f^{\prime}\left(b_{t}^{*}(b)\right)\right)>1$. It follows from (B1) that, for $\tau>t, a\left(b_{\tau-1}^{*}(b)\right) u^{\prime}\left(c_{\tau}^{*}(b)\right)$ is decreasing and $a\left(b_{\tau-1}^{*}(b)\right)$ is increasing. Hence, $u^{\prime}\left(c_{\tau}^{*}(b)\right)$ is decreasing and, by the strict concavity of $u, c_{\tau}^{*}(b)$ is increasing. This is infeasible if $\lim _{t \rightarrow \infty} b_{t}^{*}(b)=0$ by equation $(1)$ and the properties of $f$.

In case (ii), the properties of $f$ imply that there is $t \in \mathbb{N}$ such that $\alpha(1+$ $\left.f^{\prime}\left(b_{t}^{*}(b)\right)\right)<1$. It follows from (B1) that, for $\tau>t, a\left(b_{\tau-1}^{*}(b)\right) u^{\prime}\left(c_{\tau}^{*}(b)\right)$ is increasing and $a\left(b_{\tau-1}^{*}(b)\right)$ is decreasing. Hence, $u^{\prime}\left(c_{\tau}^{*}(b)\right)$ is increasing and, by the strict concavity of $u, c_{\tau}^{*}(b)$ is decreasing. Consider the feasible stream in $K\left(b_{t}^{*}(b)\right)$ with $k_{\tau}=b_{t}^{*}(b)$ for all $\tau \geq t$. It follows from equation (1) that $c_{\tau}=f\left(b_{t}^{*}(b)\right)>c_{t+1}^{*}(b) \geq c_{\tau}^{*}(b)$ for $\tau>t$, implying that $c^{*}(b)$ is inefficient. This contradicts step 3 .

Therefore, only case (iii) remains, in which case $\lim _{t \rightarrow \infty} b_{t}^{*}(b)=k_{\infty} \in(0, \infty)$ and $\lim _{t \rightarrow \infty} c_{t}^{*}(b)=f\left(k_{\infty}\right)$. By equation (B1), and the properties of $a, u$, and $f$,

$$
a\left(k_{\infty}\right) u^{\prime}\left(f\left(k_{\infty}\right)\right)=\alpha a\left(k_{\infty}\right) u^{\prime}\left(f\left(k_{\infty}\right)\right)\left(1+f^{\prime}\left(k_{\infty}\right)\right) .
$$

This implies that $\alpha\left(1+f\left(k_{\infty}\right)\right)=1$ and $k_{\infty}=k_{\infty}(\alpha)$. 


\section{Proof of Theorem 2}

By proposition 2, $\lim _{t \rightarrow \infty} b_{t}^{*}(b)=k_{\infty}(\alpha)$ and

$$
\lim _{t \rightarrow \infty} a\left(b_{t}^{*}(b)\right) u\left(c_{t}^{*}(b)\right)=a\left(k_{\infty}(\alpha)\right) u\left(f\left(k_{\infty}(\alpha)\right)\right) .
$$

As $\alpha \rightarrow 1$, it follows from the properties $a$ and $f$ that $k_{\infty}(\alpha) \rightarrow \infty, a\left(k_{\infty}(\alpha)\right) \rightarrow 0$, and $u\left(f\left(k_{\infty}(\alpha)\right)\right) \rightarrow u(\bar{c})$. Hence, as $\alpha \rightarrow 1, a\left(k_{\infty}(\alpha)\right) u\left(f\left(k_{\infty}(\alpha)\right)\right) \rightarrow 0 \cdot u(\bar{c})=0$.

\section{APPENDIX C}

\section{Proof of Proposition 3}

It follows from the envelope theorem that the partial derivative of $\hat{u}\left(k, k^{\prime}\right)$ with respect to the first variable satisfies

$$
\hat{u}_{1}\left(k, k^{\prime}\right) \geq a(\mu k) u^{\prime}(c)\left(1+f^{\prime}(\mu k+(1-\gamma)(1-\mu) k)(1-\gamma)\right)>0,
$$

and with equality if $\mu<1$, where $c=k+f(\mu k+(1-\gamma)(1-\mu) k)-k^{\prime}$. The partial derivative with respect to the second variable is given by

$$
\hat{u}_{2}\left(k, k^{\prime}\right)=-a(\mu k) u^{\prime}(c)<0 .
$$

Furthermore, if total capital is constant between the two periods, so that $k=k^{\prime}$, then $\frac{d}{d k} \hat{u}(k, k) \geq a(\mu k) u^{\prime}(f(\mu k+(1-\gamma)(1-\mu) k)) f^{\prime}(\mu k+(1-\gamma)(1-\mu) k)(1-\gamma)>0$.

This means that $\hat{u}(k, k)$ is an increasing function of $k$ with $\hat{u}(0,0)=0$. The function

$$
\tilde{u}(k)=a(b) u(b+g+f(b+(1-\gamma) g)-k)
$$

is a decreasing function of $k$ with $\tilde{u}(k)>0$ if $k=0$ and $\tilde{u}(k)=0$ if $k=b+g+$ $f(b+(1-\gamma) g)$. It follows that $\bar{k}$ defined by equation (8) - that is, $\tilde{u}(\bar{k})=\hat{u}(\bar{k}, \bar{k})$-is uniquely determined and in the interval $(0, b+g+f(b+(1-\gamma) g))$.

The proof is concluded by showing that the constant adjusted utility stream

$$
(\tilde{u}(\bar{k}), \hat{u}(\bar{k}, \bar{k}), \ldots, \hat{u}(\bar{k}, \bar{k}), \ldots)
$$

is a maximin stream. To show this, suppose that the stream given by $(\mathrm{C} 1)$ is not a maximin stream; that is, there exist $\varepsilon>0$ and an alternative $\operatorname{stream}_{1} \tilde{k}=\left(\tilde{k}_{1}, \tilde{k}_{2}, \ldots, \tilde{k}_{t}, \ldots\right)$ of total capital such that

$$
\begin{gathered}
\tilde{u}\left(\tilde{k}_{1}\right) \geq \tilde{u}(\bar{k})+\varepsilon, \\
\text { for all } t \geq 2, \hat{u}\left(\tilde{k}_{t-1}, \tilde{k}_{t}\right) \geq \hat{u}(\hat{k}, \hat{k})+\varepsilon .
\end{gathered}
$$

We show that this leads to a contradiction through a two-step proof.

Step 1: $\bar{k}>\tilde{k}_{1}>\tilde{k}_{2}>\cdots>\tilde{k}_{t-1}>\tilde{k}_{t} \cdots$. This is shown by induction. 
(Basis.) $\bar{k}>\tilde{k}_{1}>\tilde{k}_{2}$. Since $\tilde{u}(\cdot)$ is a decreasing function, it follows from (C2) that $\bar{k}>\tilde{k}_{1}$. Suppose $\tilde{k}_{1} \leq \tilde{k}_{2}$. Then, by the properties of $\hat{u}(\cdot, \cdot), \hat{u}\left(\tilde{k}_{1}, \tilde{k}_{2}\right) \leq \hat{u}\left(\tilde{k}_{1}, \tilde{k}_{1}\right)<$ $\hat{u}(\hat{k}, \hat{k})$, which contradicts (C3). Hence, $\tilde{k}_{1}>\tilde{k}_{2}$.

(Inductive step.) For all $t \geq 2$, if $\bar{k}>\tilde{k}_{t-1}>\tilde{k}_{t}$, then $\bar{k}>\tilde{k}_{t}>\tilde{k}_{t+1}$. We have that $\bar{k}>\tilde{k}_{t^{*}}$ Suppose $\tilde{k}_{t} \leq \tilde{k}_{t+1}$. Then, by the properties of $\hat{u}(\cdot, \cdot), \hat{u}\left(\tilde{k}_{t}, \tilde{k}_{t+1}\right) \leq \hat{u}\left(\tilde{k}_{t}, \tilde{k}_{t}\right)<$ $\hat{u}(\hat{k}, \hat{k})$, which contradicts (C3). Hence, $\tilde{k}_{t}>\tilde{k}_{t+1}$.

Step 2: $\lim _{t \rightarrow \infty} \hat{u}\left(\tilde{k}_{t-1}, \tilde{k}_{t}\right)=\hat{u}\left(k^{*}, k^{*}\right)<\hat{u}(\hat{k}, \hat{k})$. It follows from step 1 and the fact that $\tilde{k}_{t} \geq 0$ for all $t$ that the sequence $\tilde{k}_{1}, \tilde{k}_{2}, \ldots, \tilde{k}_{t}, \ldots$ converges to some $k^{*} \in[0, \hat{k})$. The result of step 2 is obtained by the properties of of $\hat{u}(\cdot, \cdot)$, observing in particular that this function is continuous.

The constant adjusted utility stream defined by $(\mathrm{C} 1)$ is thus a maximin stream.

\section{APPENDIX D}

\section{Proof of Theorem 4}

Consider the period $t$ asset management problem (7). Recall that, for given total capital accumulated in period $t, k$, and total capital accumulated in period $t+1, k^{\prime}$, the problem is to split $k$ into brown capital, $\mu k$, and green capital, $(1-\mu) k$, so as to achieve short-run efficiency. Assume that $a(\mu k)=e^{-\rho \mu k}$, with $\rho>0$, and that $k^{\prime} \in[0, k+f((1-\gamma) k))$ so that more than $k^{\prime}$ is attainable in period $t+1$ given $k$ in period $t$ with use of the less productive green capital only. Thus, $\mu\left(k, k^{\prime}\right)=0$ in problem (7).

With $a(\mu k)=e^{-\rho \mu k}$, the period $t$ asset management problem (7) becomes:

$$
\max _{\mu} e^{-\rho \mu k} u\left(k+f(\mu k+(1-\gamma)(1-\mu) k)-k^{\prime}\right) \text { subject to }\left\{\begin{array}{c}
-\mu \leq 0 . \\
\mu \leq 1 .
\end{array}\right.
$$

The corresponding Lagrangian is:

$$
L(\mu)=e^{-\rho \mu k} u(c)-\lambda_{1}(-\mu)-\lambda_{2}(\mu-1),
$$

where

$$
c=k+f(\mu k+(1-\gamma)(1-\mu) k)-k^{\prime} .
$$

The optimal value of $\mu, \hat{\mu}$, satisfies the first-order condition:

$$
\underbrace{-\rho k e^{-\rho \hat{\mu} k} u(c)+e^{-\rho \hat{\mu} k} u^{\prime}(c) f^{\prime}(\hat{\mu} k+(1-\gamma)(1-\hat{\mu}) k) \gamma k}_{=s(\gamma \mid \hat{\mu})}+\lambda_{1}-\lambda_{2}=0,
$$

as well as the following complementary slackness conditions:

$$
\begin{aligned}
& \lambda_{1} \geq 0, \text { with } \lambda_{1}[-\hat{\mu}]=0 . \\
& \lambda_{2} \geq 0, \text { with } \lambda_{2}[\hat{\mu}-1]=0 .
\end{aligned}
$$

It follows from the properties of $u$ and $f$ that $\lambda_{1}$ is a continuous function of $\gamma$. 
Figure D1 shows the function $s(\gamma \mid \hat{\mu})$. Recall that, for any $\rho>0$, we seek the highest possible $\gamma(\rho)$ ensuring that problem (D1) is solved for the boundary solution $\hat{\mu}=0$ rather than an interior solution or the boundary solution $\hat{\mu}=1$. Therefore $\lambda_{2}=0$.

Set $\hat{\mu}=0$ and $\lambda_{2}=0$ in equation (D2), and let $\lambda_{1}$ approach 0 :

$$
\begin{array}{r}
\lim _{\lambda_{1} \rightarrow 0}\left[-\rho k u(c)+u^{\prime}(c) f^{\prime}((1-\gamma) k) \gamma k+\lambda_{1}\right] \\
=-\rho k u(c)+u^{\prime}(c) f^{\prime}((1-\gamma) k) \gamma k=0 .
\end{array}
$$

Rearranging terms, we can define $\gamma(\rho)$, for any $\rho>0$, by

$$
\rho \frac{u(c)}{u^{\prime}(c) f^{\prime}((1-\gamma(\rho)) k) \gamma(\rho)}=1 .
$$

It follows from the properties of $u$ and $f$ and the assumption that $k^{\prime} \in[0, k+f(k))$ that $\gamma(\rho)$ is well defined, continuous, and increasing, with $\lim _{\rho \rightarrow 0} \gamma(\rho)=0$. For any $\rho>0, \gamma(\rho)$ is the highest possible value of $\gamma$ consistent with short-run efficiency in all periods in the case where only green capital is accumulated.

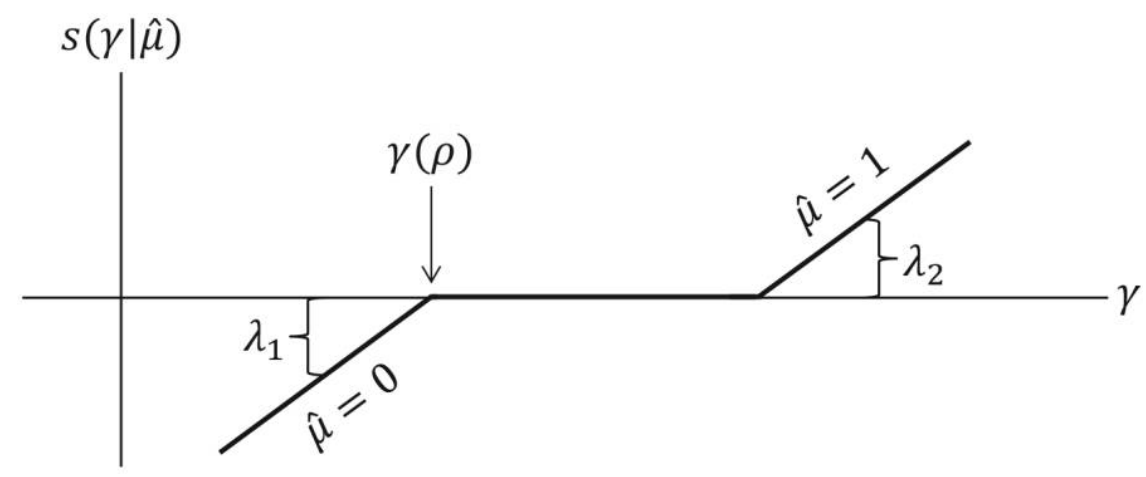

Figure D1. $s(\gamma \mid \hat{\mu})$, with $\hat{\mu}$ indicating optimal $\mu$

\section{REFERENCES}

Asheim, Geir B. 2008. Paradoxical consumption behavior when economic activity has environmental effects. Journal of Economic Behavior and Organization 65:529-46.

2010. Intergenerational equity. Annual Review of Economics 2:197-222.

Asheim, Geir B., Wolfgang Buchholz, and Bertil Tungodden. 2001. Justifying sustainability. Journal of Environmental Economics and Management 41:252-68.

Asheim, Geir B., and Tapan Mitra. 2010. Sustainability and discounted utilitarianism in models of economic growth. Mathematical Social Sciences 59:148-69.

Basu, Kaushik, and Tapan Mitra. 2007. Utilitarianism for infinite utility streams: A new welfare criterion and its axiomatic characterization. Iournal of Economic Theory 133:350-73. 
Beals, Richard, and Tjalling C. Koopmans. 1969. Maximizing stationary utility in a constant technology. SIAM Journal of Applied Mathematics 17:1001-15.

Calvo, Guillermo. 1978. Some notes on time inconsistency and Rawls' maximin criterion. Review of Economic Studies 45:97-102.

Farzin, Y. Hossein. 1984. The effect of the discount rate on the depletion of natural resources. $\underline{\text { Journal of }}$ Political Economy 92:841-51.

Foley, Duncan K. 2008. The economic fundamentals of global warming. In Twenty-first century macroeconomics: Responding to climate challenge, ed. Jonathan M. Harris and Neva R. Goodwin. Northampton: Edward Elgar.

Government of Norway. 2016. Høring-NOU 2012 16: Samfunnsøkonomiske analyser. https://www .regjeringen.no/no/dokumenter/horing—nou-201216-samfunnsokonomiske-a/id701086/.

Howarth, Richard B., and Richard B. Norgaard. 1995. Intergenerational choice under global environment change. In Handbook of environmental economics, ed. Daniel W. Bromley. Oxford: Blackwell.

John, A., and R. Pecchenino. 1994. An overlapping generations model of growth and the environment. Economic Journal 104:1393-1410.

Jouvet, Pierre-André, Philippe Michel, and Jean-Pierre Vidal. 2000. Intergenerational altruism and the environment. Scandinavian Journal of Economics 102:135-50.

Karp, Larry. Forthcoming. Provision of a public good with multiple dynasties. Economic Journal.

Lauwers, Luc. 2010. Ordering infinite utility streams comes at the cost of a non-Ramsey set. Iournal of Mathematical Economics 46:32-37.

Michel, Philippe, Emmanuel Thibault, and Jean-Pierre Vidal. 2006. Intergenerational altruism and neoclassical growth models. In Handbook of the economics of giving, altruism and reciprocity, vol. 2, ed. SergeChristophe Kolm and Jean Mercier Ythier. Amsterdam: Elsevier.

Nordhaus, William. 2008. A question of balance: Weighting the options on global warming policies. New Haven, CT: Yale University Press.

Ray, Debraj. 1987. Nonpaternalistic intergenerational altruism. Iournal of Economic Theory 41:112-32.

Rezai, Armon, Duncan K. Foley, and Lance Taylor. 2012. Global warming and economic externalities. Economic Theory 49:329-51.

Saez-Marti, Maria, and Jörgen W. Weibull. 2005. Discounting and altruism to future decision-makers. Journal of Economic Theory 122:254-66.

Sen, Amartya K. 1970. Collective choice and social welfare. Edinburgh: Oliver \& Boyd.

Sierra Club. 2016. Energy economics. http://www.sierraclub.org/policy/energy/energy-economics.

Suppes, Patrick. 1966. Some formal models of grading principles. Synthese 6:284-306.

Zame, William R. 2007. Can intergenerational equity be operationalized? Theoretical Economics 2:187-202.

Zuber, Stéphane, and Geir B. Asheim. 2012, Justifying social discounting: The rank-discounted utilitarian approach. Iournal of Economic Theory 147:1572-1601. 